\title{
Change Detection in Water reservoir using Image Fusion and Supervised Classifier
}

\author{
Kiran Khandarkar ${ }^{1}$, Dr Sharvari Tamne ${ }^{2}$ \\ ${ }^{1}$ Assistant Professor, Maharashtra Institute of Technology, Aurangabad, Maharashtra, India \\ ${ }^{2}$ Professor and Head IT Department, MGMs JNEC Aurangabad, Maharashtra, India
}

\begin{abstract}
Article Info

Volume 7, Issue 6

Page Number : $324-330$

Publication Issue :

November-December-2021

Article History

Accepted : 12 Dec 2021

Published : 26 Dec 2021

The research provides a method for improving change detection in SAR images using a fusion object and a supervised classification system. To remove noise from the input image, we use the DnCNN denoising approach. The data from the first image is then processed with the mean ratio operator. The log ratio operator is used to process the second image. These two images are fused together using SWT-based image fusion, and the output is sent to a supervise classifier for change detection.

Keywords : SAR, difference-image, image-fusion, CDA, DnCNN.
\end{abstract}

\section{INTRODUCTION}

Remote sensing-technology's most important application is identification of changes occurring on the surface of earth using multi-temporal remote sensing images. Change detection usually involves examining two co-registered remote sensing images that were collected at different time instances over the same geographic area. Such an approach is considered unattended when it is meant to discriminate between two different groups without any experience of the scene beforehand. Different automated and unsupervised methods of change detection are developed and described in the literature of remote sensing. Unsupervised-ChangeDetection in SAR images can be considered as having three different modules such as image pre-processing, Producing change in image, and analysis of that change in image [2]
A difference image will be generated using complimentary information from a mean-ratio image and a log-ratio image using the image fusion approach. For a low-frequency band and a high-frequency band, SWT (Stationary Wavelet transform) fusion criteria based on an average operator and minimum local area Energy are used. Subtraction operator and rationing are well-known approaches for obtaining a difference image in remote sensing photographs. For categorising modified and unchanged regions in the fused difference image, an artificial neural network type multi layer perceptron or back propagation with feed forward network will be proposed. This classifier belongs to the supervised segmentation category, and it operates on the basis of training cum classification. The results showed that rationing produces a better difference image for change detection when utilizing a supervised classifier segmentation approach and the 
algorithm's effectiveness will be measured by its accuracy and specificity [16][20][21].

In the first step, input images are denoised by CNN denoising algorithm. The second stage involves applying a pixel-by-pixel mean-ratio and log-ratio operator on the pair of temporal images. SWT (Stationary Wavelet transform)[20][21] fusion based on an average operator and minimum local area energy is performed in the third step, To restrict background information and improve the information of changed regions in the fused difference picture. In the fourth stage, image classification using supervised learning using a non-knowledge based classifier is employed to segregate the changes in region from the fused image. For SAR imaging, shift-detection techniques are developed using Seasat SAR observations for the temporal monitoring of multiyear sea-ice floes. Changes in detection techniques can be classified into several SAR image groups, each corresponding to specific image quality specifications. Changes are found in a first category based on temporary object tracking or stable geometric form recognition image characteristics. In many applications for signal and image processing, the combination of information acquired from multiple sensors has become very common. For earth observation applications, data provided by different sensor-types provides a complement that improves the limitations of a particular type-of-sensor. User must use the photos available in the database or the first acquisition available after an interest case. Both image capture and alteration detection strategies consist of comparing more than one picture of reference image and second image obtained over the target scene at two distinct dates with same time. The reference image is usually retrieved from a database and the second image processing is planed after a mark-able change[15].

\section{PROPOSED TECHNIQUE}

The objective of the proposed technique is to detect and locate changes in two SAR images with Back propagation feed forward neural network model, using stationary wavelet-based transform fusion

In this proposed method, five modules are used Image Denoising, Difference Generation, Differenced Image Fusion, Supervised Image Segmentation and Performance analysis.

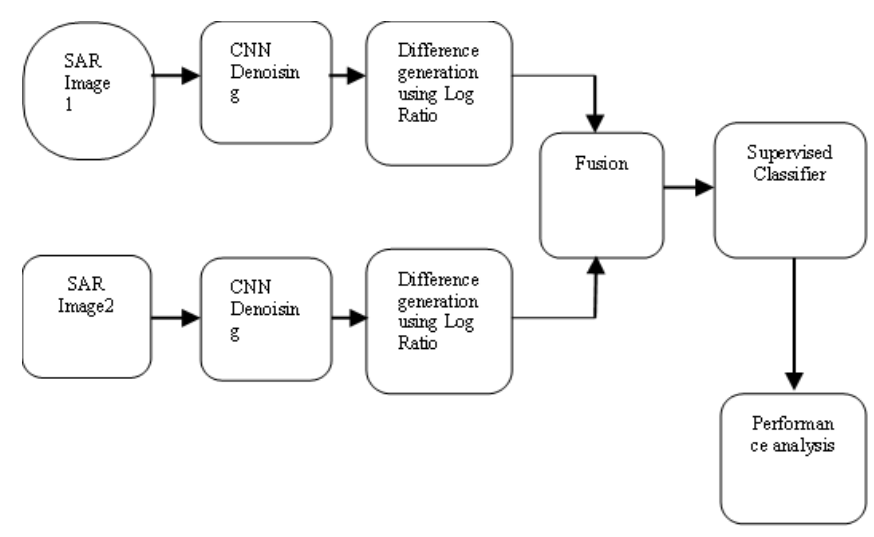

Fig.1 System architecture.

Input image denoising

Input SAR images are denoised using (DnCNN) deep neural network denoising algorithm. If noise is present in the input images then it can affect the change detection in SAR images. So to overcome this issue we had used Denoising technique on SAR images.

\section{Difference Image Creation}

To get difference images in logarithmic and mean scales, rationing is used. It is highly robust to speckle noise.

To detect changes and unchanging regions, a logarithmic scale based difference portion will be developed, but it will weaken the high intensity pixels[20][21] while enhancing the low intensity pixels. There is a risk of considerable information loss as a result of this deterioration. As a result, a ratio mean operator and fusion approach are utilized to overcome this constraint and synthesize detailed 
portions from source images in order to detect precise alterations [15].

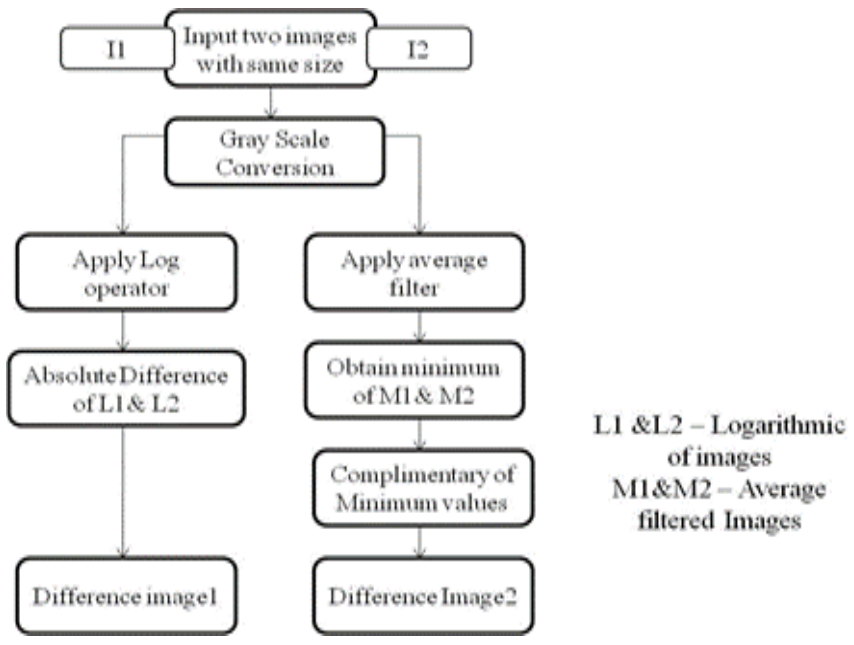

Fig. 2. Difference image generation process flow

In first step DI are generated by following formula,

Log-ratio:

$$
\mathrm{DI} 1=|\log \mathrm{Y} 2-\log \mathrm{Y} 1|
$$

Where,

Y2 - Input picture1 and Y1 - Input picture 2

Mean_ratio:

$$
\mathrm{DI} 2=1-\min (\mathrm{v} 1 / \mathrm{v} 2, \mathrm{v} 2 / \mathrm{v} 1) \text {; }
$$

Where,

v1 - average-filtered-image1, v2 - average-filteredimage

Image Fusion

The morphing procedure is used to retrieve the enhanced details about the changing region from the unchanged region using the sub-band images of two source images obtained from SWT.

High-frequency components are fused using energybased fusion, whereas low-frequency components are mixed using the averaging method. Then output of these two methods is provided to Inverse SWT to produce final fused image.

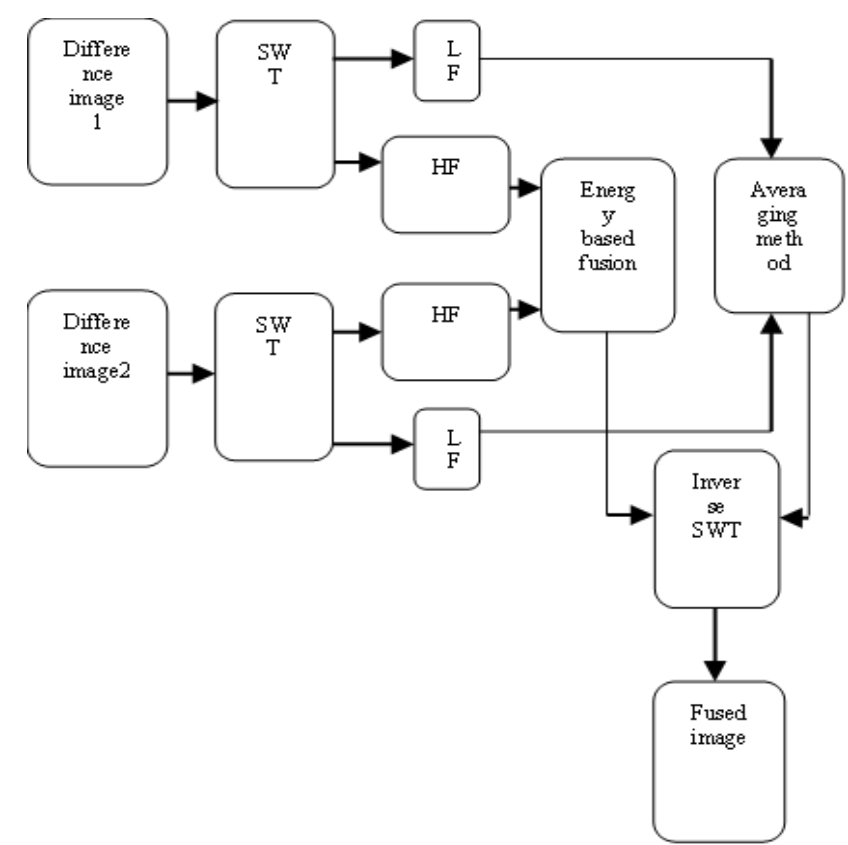

Fig.3 Fusion Design model

\section{STUDY AREA DETAILS}

"Nathsagar Water dam" situated in Paithan Village is used as a study area for my research. The coordinates of the study area are Lat 19.515' lon 75.27'

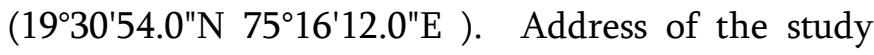
area is Village Paithan, Dist Aurangabad, State Maharashtra , India.

Time period of satellite Images taken of the study area are, from 10th January 2019 to 12th March 2021.

Satellite images are taken from Copernicus Sentinel data 2021 Retrieved from ASF DAAC 10th January to 12th march 2021, processed by ESA [9].

\section{Details about satellite used:}

Sentinel-1 1 C-Band

Start Time $\cdot 03 / 12 / 21,00: 54: 42 Z$

Stop Time $\cdot 03 / 12 / 21,00: 55: 07 Z$

Beam Mode $\cdot$ IW

Path $\cdot 136$

Frame $\cdot 526$

Flight Direction $\cdot$ DESCENDING

Polarization $\cdot \mathrm{VV}+\mathrm{VH}$

Absolute Orbit $\cdot 36958$ 
Data courtesy of ESA.

\section{EXPERIMENTS AND RESULTS}

To assess the proposed method's performance, we use three different data sets with varied features to test it. The first data set [13] is a segment (301X 301 pixels) of two SAR images collected by the European Remote Sensing 2 satellite SAR sensor in April and May 1999, respectively, over an area near Bern, Switzerland. Between the two days, the River Aare completely inundated areas of the cities of Thun and Bern, as well as the Bern airport. The Ottawa data set[12][19], which shows a part of two SAR images obtained by a RADARSAT SAR sensor, is the second data set used. The size of the images is $290 \times 350$ pixels. The National Defense Research and Development Canada contributed the data[19]. The photographs depict the places that were formerly flooded. The third data set is the Yellow River data set. It shows a portion of SAR photos taken by Radarset-2 in the Yellow River Estuary region of China[17].

I had used total 30 images of the study area and standard dataset of Bern, Ottawa, and yellow river for experimentation. For performance evaluation I used sensitivity, specificity, accuracy and Pearson's Correlation Coefficient.

Sensitivity and specificity: These are statistical measures that are used to evaluate a binary classification test's performance. The fraction of accurately detected positives is measured by sensitivity [10] (True Positive rate). The proportion of water that has changed (affected) and has been appropriately detected as having altered. The proportion of accurately detected negatives is measured by specificity (True Negative Rate). The proportion of water that has not changed and has been appropriately detected as not being in the change condition.

The terms TP, TN, FN, and FP are used to describe sensitivity, specificity, and accuracy.

Sensitivity $=\mathrm{TP} /(\mathrm{TP}+\mathrm{FN})=($ Number of true positive assessments)/(Number of all positive assessment)
Specificity $=\mathrm{TN} /(\mathrm{TN}+\mathrm{FP})=$ (Number of true negative assessments)/ (Number of all negative assessment)

Accuracy $=(\mathrm{TN}+\mathrm{TP}) /(\mathrm{TN}+\mathrm{TP}+\mathrm{FN}+\mathrm{FP})=($ Number of correct assessments)/(Number of all assessments).

The sensitivity numbers values show the likelihood that a test will identify images with the modification. The higher the sensitivity numerical numbers, the less likely a diagnostic test may produce false-positive results. If sensitivity $=99$ percent, for example, it means that when we perform a diagnostic test on a test image with a specific change, there is a 99 percent chance that the change will be classified as positive. A test using high-sensitivity tents to capture all possible positive situations while ensuring that no one is left out. In medical science, a test with a high sensitivity is frequently employed to screen for disease. Specificity is a numerical value that shows the likelihood that a test would correctly diagnose a specific picture without producing false-positive findings. For instance, if a test's specificity is 99 percent, it indicates that when we run a diagnostic test on a picture that hasn't changed, there's a 99 percent likelihood that the image will be classified as negative.

The proportion of true positive outcomes (both true positive and true negative) in the selected population is represented by the numerical value of accuracy. The test result is accurate 99 percent of the time, whether it is positive or negative and this is true for most of the cases.

\section{Pearson's Correlation Coefficient (PCC):}

In statistical analysis, pattern recognition, and image processing, Pearson's method[11] is commonly employed. The latter has applications such as comparing two images for image registration and disparity measurement. It is described in

$$
r_{1}=\frac{\sum_{i}\left(x_{i}-x_{m}\right)\left(y_{i}-y_{m}\right)}{\sqrt{\sum_{i}\left(x_{i}-x_{m}\right)^{2}} \sqrt{\sum_{i}\left(y_{i}-y_{m}\right)^{2}}}
$$


Dataset and Criteria for Evaluation Where xi is the ith pixel's intensity in image 1 , yi is the ith pixel's intensity in image $2, \mathrm{xm}$ is the mean intensity of image 1 , and $y m$ is the mean intensity of image 2 . The correlation coefficient is $r=1$ if the two images are totally identical, $\mathrm{r}=0$ if they are fully uncorrelated, and $\mathrm{r}=-1$ if they are completely anti-correlated, such as if one image is the inverse of the other[18].

TABLE I

QUALITY EVALUATION ON DIFFERENT DATASETS

\begin{tabular}{|l|l|l|l|l|}
\hline Data set & OE \% & $\begin{array}{l}\text { PCC } \\
\%\end{array}$ & Specificity & Accuracy \\
\hline Bern & 0.686645508 & 99.31 & 99.45 & 99.31335 \\
\hline Ottawa & 11.50665283 & 88.49 & 89.12 & 88.49335 \\
\hline Farmland & 9.959411621 & 90.04 & 95.42 & 90.04059 \\
\hline $\begin{array}{l}\text { Study } \\
\text { Area }\end{array}$ & 4.531860352 & 95.46 & 98.12 & 95.46814 \\
\hline
\end{tabular}

TABLE III

PERFORMANCE EVALUATION ON FARMLAND REAL SAR IMAGE DATASETS

\begin{tabular}{|l|l|l|l|l|l|}
\hline Method & PFA & PMD & OE & PCC & KC \\
\hline PCAKM[14] & 3.51 & 19.83 & 2.24 & 93.54 & 77.85 \\
\hline MRFFCM & 1.05 & 22.14 & 2.89 & 95.13 & 82.38 \\
\hline GaborTLC & 1.9 & 17.21 & 3.2 & 95.33 & 83.68 \\
\hline D_MRFFCM & 1.08 & 19.92 & 3.23 & 95.51 & 83.92 \\
\hline $\begin{array}{l}\text { Feng Gao, } \\
\text { Junyu Dong } \\
\text { 2016 }\end{array}$ & 3.1 & 10.62 & 3.62 & 95.54 & 85.15 \\
\hline
\end{tabular}

Fig 8. Screenshots of Experiment execution on study area

\section{CONCLUSION}

We introduce a novel SAR-image change detection strategy[22] based on picture fusion and a supervised classifier algorithm in this research, which differs from existing methods. We employed the DnCNN denoising approach to remove noise from the input image, which enhances the overall system's accuracy. The fusion approach for merging two photos obtained at separate times to increase features of changed 
region from unchanging region was used to detect changed region. I had used total 30 images of the study area and standard dataset of Bern, Ottawa, and yellow river. With our experimentation we got $95 \%$ accuracy on study area and $98 \%$ accuracy on Bern dataset.

\section{REFERENCES}

[1]. Qian Wei, Yaoguo Zheng, and Shuang Wang, Biao Hou, Member, Member, IEEE "Unsupervised Change Detection in SAR Image Based on Gauss-Log Ratio Image Fusion and Compressed Projection", IEEE journal of selected topics in applied earth observations and remote sensing, vol. 7, no. 8, august 2014.

[2]. K. R. Khandarkar, Dr. A. N. Holambe, "Survey on Change Detection in SAR Images with Image Fusion and Image Segmentation “, International journal of scientific research in science \& Technology, Feb 2018.

[3]. Hire Gayatri Ashok , D. R. Patil " Survey on Change Detection in SAR Images ", National Conference on Emerging Trends in Computer Technology (NCETCT),2014.

[4]. V. Petrovic , T. Cootes "Information Representation for Image Fusion Evaluation" 9th International Conference on Information Fusion, 2006.

[5]. Francesca Bovolo, Lorenzo Bruzzone "The Time Variable in Data Fusion: A Change Detection Perspective" , IEEE Geoscience and remote sensing magazine, September 2015.

[6]. Hire Gayatri Ashok, D. R. Patil "Image Fusion and Fuzzy Clustering based Change Detection in SAR Images", International Conference on Advances in Science and Technology (ICAST2014)

[7]. K. Venkateswaran, N. Kasthuri, Arathy.C.Haran.V "Unsupervised Change Detection using Image Fusion and Kernel KMeans Clustering" , International Conference on Innovations In Intelligent Instrumentation, Optimization And Signal Processing"ICIIIOSP2013.

[8]. M.Adithyan , R.Arul Pandi , A.Ramesh, J.Venkatesen " Dual Ratio Operator and Wavelet Fusion Based Supervised Change Detection in SAR Images " International Journal of Engineering science and computing, Volume 6 Issue No. 4 ,2016.Lu Jia,Ming Li,Peng Zhang,Yan Wu,Lin An,and Wanying Song " Remote-Sensing Image

[9]. Copernicus Sentinel data 2021 Retrieved from ASF DAAC 10th January to 12th march 2021, processed by ESA.

[10]. Wen Zhu, Nancy Zeng, Ning Wang "Sensitivity, Specificity, Accuracy, Associated Confidence Interval and ROC Analysis with Practical SAS® Implementations" NESUG 2010 Health Care and Life Sciences.

[11]. A. Miranda Neto, A. Correa Victorino, I. Fantoni, D. E. Zampieri, J. V. Ferreira and D. A. Lima “ Image Processing Using Pearson's Correlation Coefficient: Applications on Autonomous Robotics " 13th International Conference on Autonomous Robot Systems IEEE 2013.

[12]. Yunhao Gao, Feng Gao , Junyu Dong , and Shengke Wang “ Change Detection From Synthetic Aperture Radar Images Based on Channel Weighting-Based Deep Cascade Network “,IEEE JOURNAL OF SELECTED TOPICS IN APPLIED EARTH OBSERVATIONS AND REMOTE SENSING , 2019.

[13]. Maoguo Gong, Member, IEEE, Zhiqiang Zhou, and Jingjing $\mathrm{Ma}$ "Change Detection in Synthetic Aperture RadarImages based on Image Fusion and Fuzzy Clustering" IEEE TRANSACTIONS ON IMAGE PROCESSING, VOL. 21, NO. 4, APRIL 2012

[14]. Feng Gao, Junyu Dong, Bo Li, and Qizhi Xu , "Automatic Change Detection in Synthetic 
Aperture Radar Images Based on PCANet", IEEE.

[15]. G.Shanthana Bharathi, B.Pandeeswari "Change Detection in SAR Images Using Intensity and Texture Features" International Journal of Engineering Science and Computing, ISSN 2321 3361 @ 2016 IJESC, June 2016.

[16]. MerinAyshu Ali , DR. B. M. Imran "An Efficient Approach for Change Detection in SAR Images Based on Contourlet Fusion", IJAREEIE , Vol. 2, Special Issue 1, December 2013 , ISSN (Online): 2278 - 8875.

[17]. Xiao Wang, Feng Gao, Junyu Dong and Shengke Wang "Sea Ice Change Detection from SAR Images Based on Canonical Correlation Analysis and Contractive Autoencoders" Springer Nature Switzerland AG 2018 R. Hong et al. (Eds.): PCM 2018, LNCS 11165, pp. 748757, 2018.

[18]. Maria Tatesa,b, Nasser M. Nasrabadia , Heesung Kwona "Locating mines in SAR imagery using change detection methods", Proceedings of the 5th WSEAS International Conference on Signal Processing, Istanbul, Turkey, May 27-29, 2006 (pp24-29).

[19]. GeoAI 2019: Proceedings of the 3rd ACM SIGSPATIAL International Workshop on AI for Geographic Knowledge DiscoveryNovember 2019

[20]. MerinAyshu Ali, DR. B. M. Imran "An Efficient Approach for Change Detection in SAR Images Based on Contourlet Fusion" International Journal of Advanced Research in Electrical, Electronics and Instrumentation Engineering Vol. 2, Special Issue 1, December 2013

[21]. Krishna Kant Singh1 , Neelima Sain "Unsupervised Change Detection for Satellite Images using Normalized Neighborhood Ratio and Gustafson KesselClustering” International Journal of Advanced Research in Electrical, Electronics and Instrumentation Engineering, Vol. 2, Issue 11, November 2013.
[22]. K.R. Khandarkar, Sharvari C. Tamane "Change Detection in SAR Images using Image Fusion and Supervised Classifier" , International Journal of Recent Technology and Engineering (IJRTE) ISSN: 2277-3878, Volume-9 Issue-1, May 2020.

\section{Cite this article as :}

Kiran Khandarkar, Dr. Sharvari Tamne, "Change Detection in Water reservoir using Image Fusion and Supervised Classifier", International Journal of Scientific Research in Computer Science, Engineering and Information Technology (IJSRCSEIT), ISSN : 2456-3307, Volume 7 Issue 6, pp. 324-330, November-December 2021. Available at doi : https://doi.org/10.32628/CSEIT217685 Journal URL : https://ijsrcseit.com/CSEIT217685 